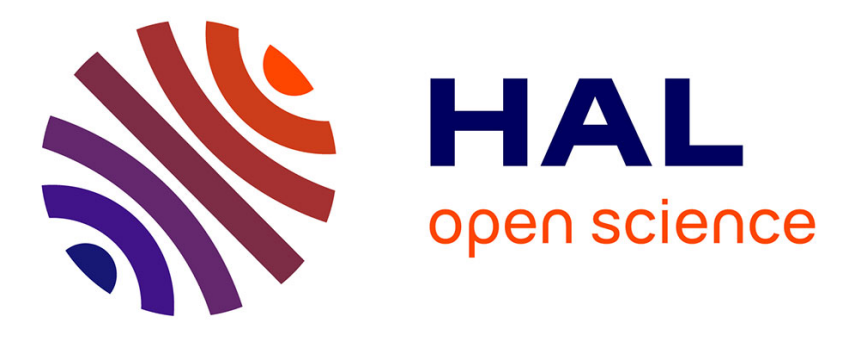

\title{
_ $28^{68} \mathbf{N i} \_40:$ Magicity versus superfluidity
}

O. Sorlin, S. Leenhard, C. Donzaud, J. Duprat, F. Azaiez, F. Nowacki, H.

Grawe, Zs. Dombradi, F. Amorini, A. Astier, et al.

\section{To cite this version:}

O. Sorlin, S. Leenhard, C. Donzaud, J. Duprat, F. Azaiez, et al.. _ $28^{68} \mathrm{Ni}$ 40: Magicity versus superfluidity. Physical Review Letters, 2002, 88, pp.092501-1-092501-5. 10.1103/PhysRevLett.88.092501. in2p3-00013604

\section{HAL Id: in2p3-00013604 https://hal.in2p3.fr/in2p3-00013604}

Submitted on 21 May 2021

HAL is a multi-disciplinary open access archive for the deposit and dissemination of scientific research documents, whether they are published or not. The documents may come from teaching and research institutions in France or abroad, or from public or private research centers.
L'archive ouverte pluridisciplinaire HAL, est destinée au dépôt et à la diffusion de documents scientifiques de niveau recherche, publiés ou non, émanant des établissements d'enseignement et de recherche français ou étrangers, des laboratoires publics ou privés. 


\section{${ }_{28}^{68} \mathrm{Ni}_{40}$ : Magicity versus Superfluidity}

O. Sorlin, ${ }^{1}$ S. Leenhardt, ${ }^{1}$ C. Donzaud,${ }^{1}$ J. Duprat, ${ }^{1}$ F. Azaiez, ${ }^{1}$ F. Nowacki, ${ }^{2}$ H. Grawe,${ }^{3}$ Zs. Dombrádi, ${ }^{4}$ F. Amorini, ${ }^{5}$ A. Astier, ${ }^{6}$ D. Baiborodin, ${ }^{7}$ M. Belleguic, ${ }^{1}$ C. Borcea,${ }^{8}$ C. Bourgeois, ${ }^{1}$ D. M. Cullen,,${ }^{9} *$ Z. Dlouhy, ${ }^{7}$ E. Dragulescu, ${ }^{8}$ M. Górska, ${ }^{3}$ S. Grévy, ${ }^{10}$ D. Guillemaud-Mueller, ${ }^{1}$ G. Hagemann, ${ }^{11}$ B. Herskind, ${ }^{11}$ J. Kiener, ${ }^{12}$ R. Lemmon, ${ }^{13}$ M. Lewitowicz, ${ }^{14}$ S. M. Lukyanov, ${ }^{15}$ P. Mayet,${ }^{3}$ F. de Oliveira Santos, ${ }^{14}$ D. Pantalica, ${ }^{7}$ Yu.-E. Penionzhkevich, ${ }^{15}$ F. Pougheon, ${ }^{1}$ A. Poves,${ }^{16}$ N. Redon, ${ }^{6}$ M. G. Saint-Laurent, ${ }^{14}$ J. A. Scarpaci, ${ }^{1}$ G. Sletten, ${ }^{11}$ M. Stanoiu, ${ }^{14}$ O. Tarasov, ${ }^{15, \dagger}$ and Ch. Theisen ${ }^{17}$

${ }^{1}$ Institut de Physique Nucléaire, IN2P3-CNRS, F-91406 Orsay Cedex, France

${ }^{2}$ IReS, IN2P3-CNRS, Université Louis Pasteur, BP 28, F-67037 Strasbourg Cedex, France

${ }^{3}$ GSI, Postfach 110552, D-64200 Darmstadt, Germany

${ }^{4}$ Institute of Nuclear Research, H-4001 Debrecen, Pf. 51, Hungary

${ }^{5}$ INFN-Laboratori Nazionali del Sud, via S. Sofia 44, I-95123 Catania, Italy

${ }^{6}$ Institut de Physique Nucléaire Lyon, F-69622 Villeurbanne Cedex, France

${ }^{7}$ Nuclear Physics Institute, AS CR, CZ 25068, Rez, Czech Republic

${ }^{8}$ IFIN-HH, P.O. Box MG-6, ROM-76900 Bucarest-Magurele, Romania

${ }^{9}$ Oliver Lodge Laboratory, University of Liverpool, Liverpool L69 7ZE, United Kingdom

${ }^{10}$ LPC, ISMRA, F-14050 Caen Cedex, France

${ }^{11}$ NBI, University of Copenhagen, Copenhagen, Denmark

${ }^{12}$ CSNSM, F-91405 Orsay Cedex, France

${ }^{13}$ CRLC, Daresbury Laboratory, Daresbury, Warrington WA4 4AD, United Kingdom

${ }^{14}$ GANIL, BP 5027, F-14076 Caen Cedex, France

${ }^{15}$ FLNR, JINR, 141980 Dubna, Moscow region, Russia

${ }^{16}$ Departamento de Fisica Teorica, Universidad Autónoma de Madrid, Cantoblanco, ES-28049 Madrid, Spain

${ }^{17}$ CEA-Saclay, DAPNIA-SPhN, F-91191 Gif sur Yvette Cedex, France

(Received 21 December 2000; revised manuscript received 15 November 2001; published 15 February 2002)

The neutron-rich ${ }^{66,68} \mathrm{Ni}$ have been produced at GANIL via interactions of a $65.9 \mathrm{~A} \mathrm{MeV}{ }^{70} \mathrm{Zn}$ beam with a ${ }^{58} \mathrm{Ni}$ target. Their reduced transition probability $B\left(E 2 ; 0_{1}^{+} \rightarrow 2^{+}\right)$has been measured for the first time by Coulomb excitation in a ${ }^{208} \mathrm{~Pb}$ target at intermediate energy. The $B(E 2)$ value for ${ }^{68} \mathrm{Ni}_{40}$ is unexpectedly small. An analysis in terms of large scale shell model calculations stresses the importance of proton core excitations to reproduce the $B(E 2)$ values and indicates the erosion of the $N=40$ harmonic-oscillator subshell by neutron-pair scattering.

DOI: $10.1103 /$ PhysRevLett.88.092501

PACS numbers: 21.10.Tg, 25.70.Gh, 27.50.+e

The search for an understanding of how shell structure develops is a major goal in the study of quantum systems. In atomic systems, closed-shell atoms, i.e., rare gases, are inert because of their high electronic ionization potential. In alkali clusters, the electronic structure is dominated by the number of valence electrons; closed-shell clusters exhibit spherical shapes and higher stability. In hot stellar environments such as those encountered in type II supernovae, magic nuclei are the main "survivors" and play a decisive role in the fate of the exploding stars as well as in the nucleosynthesis of new elements.

Nuclear systems can be distinguished from atomic ones by two remarkable properties which have important consequences for the location of closed shells. First, nuclei are composed of two fluids, protons and neutrons. Magic numbers can therefore be found for both protons and neutrons, leading to doubly magic nuclei. Second, the nuclear interaction is spin dependent. A strong spin-orbit interaction is required to model nuclei. This term is expected to lower the $l+1 / 2$ orbital. A direct consequence of the lowering of the $(l=4) g_{9 / 2}$ orbital is the reduction of the gap at the neutron number $N=40$, shifting the magic number to $N=50$. Nuclei close to stability, therefore, exhibit a sequence of magic numbers of $2,8,20,28,50,82, \ldots$, which are different from those calculated with harmonic oscillator potential: $2,8,20,40,70, \ldots$.

Experimental evidence for vanishing of shell effects at $N=20$ and $N=28$ for nuclei with large neutron excess have been found already by many authors. The neutronproton interaction is responsible for changes in the singleparticle orbital spacings which subsequently modifies the sizes of the shell gaps [1]. For medium-mass neutron-rich nuclei, it is expected that their surface would be essentially composed of a diffuse neutron matter [2]. As a consequence, the derivative of the mean-field potential is thought to be weaker, which reduces the spin-orbit surface term. This leads to a restoring of the gap at $N=40$ and a reduction of that at $N=50$, allowing the description of these nuclei in terms of harmonic oscillator (HO) potentials. Indeed, early mean-field calculations have predicted a distinct shell gap for the $N=40$ nucleus ${ }^{68} \mathrm{Ni}$ [3], which disappears when quadrupole correlations are taken into account $[4,5]$. 
A specific feature of $\mathrm{HO}$ numbers is that a change of parity occurs across the shell gap. It is, therefore, expected that excitations which preserve the parity symmetry across the gap, such as quadrupole excitations $E 2$, would be strongly hindered. In order to search for the magicity at $N=40$ with a moderately high neutron excess, studies of the ${ }_{28}^{68} \mathrm{Ni}_{40}$ nucleus have been made by several authors using the new techniques afforded by radioactive ion beams [6-11]. Even though the observation of the Ni isotopes spans from ${ }_{28}^{48} \mathrm{Ni}_{20}$ [12] to ${ }_{28}^{78} \mathrm{Ni}_{50}$ [13], $B\left(E 2 ; 0^{+} \rightarrow 2^{+}\right)$ measurements were hitherto limited to nuclei between ${ }^{56} \mathrm{Ni}$ and ${ }^{64} \mathrm{Ni}$. We report here the results of the measurements of the quadrupole excitation probability $B\left(E 2 ; 0^{+} \rightarrow 2^{+}\right)$ of ${ }_{28}^{66,68} \mathrm{Ni}_{38,40}$ and discuss the consequence of this new information with respect to a $\mathrm{HO}$ shell gap at $N=40$.

The experiment was performed at the GANIL facility using a $65.9 \mathrm{~A} \mathrm{MeV},{ }^{70} \mathrm{Zn}^{29+}$ beam with an average intensity of $400 \mathrm{nA}$. This primary beam was first employed with a reduced intensity to confirm the known $B(E 2)$ values of ${ }^{70} \mathrm{Zn}$ for use as a reference to determine that of ${ }^{66,68} \mathrm{Ni}$. The maximum intensity beam was subsequently used for the production of the radioactive secondary ${ }^{66,68} \mathrm{Ni}$ beams via reactions in a $140 \mu \mathrm{m}$ thick ${ }^{58} \mathrm{Ni}$ target. Nuclei of interest have been selected by the LISE3 spectrometer [14]. Mean rates of 18000 per second have been obtained for both ${ }^{66} \mathrm{Ni}$ and ${ }^{68} \mathrm{Ni}$ isotopes. Coulomb excitation of these two nuclei and ${ }^{70} \mathrm{Zn}$ at $v / c \sim 0.3$ was induced by a ${ }^{208} \mathrm{~Pb}$ target of $220 \mathrm{mg} / \mathrm{cm}^{2}$ thickness located at the final focal plane of LISE3. The target was surrounded by four segmented clover Ge detectors [15] placed at $90^{\circ}$ at a distance of $5.5 \mathrm{~cm}$ of the target, yielding a photopeak efficiency of $\epsilon_{\gamma}=4.0 \%$ at $1.3 \mathrm{MeV}$.

Two $x-y$ sets of parallel plate avalanche counters and drift chambers were mounted $40 \mathrm{~cm}$ before and after the $\mathrm{Pb}$ target, in order to track the directions of the incident nuclei prior to and after their interaction in the target. The beam profiles of incoming ${ }^{70} \mathrm{Zn}$ and of ${ }^{66,68} \mathrm{Ni}$ were very similar. This ensures that the Coulomb excitation of all nuclei has been achieved under the same geometrical conditions. Two annular silicon detectors were mounted $50 \mathrm{~cm}$ behind the lead target in order to identify the deflected nuclei by their energy losses and residual energies. They covered geometrical angles from $1.5^{\circ}$ to $6.5^{\circ}$ in the reference frame of the projectiles. Nuclei emerging from the target with smaller angles were detected in a plastic scintillator $2 \mathrm{~m}$ downstream. This detector also served to monitor the secondary beam intensity and determine the total number of implanted nuclei, $N_{n}$. The isomeric beam content from the $0_{2}^{+}$state with $t_{1 / 2}=270(5) \mathrm{ns}$, as remeasured in the present experiment, and the $5^{-}, t_{1 / 2}=0.86(5) \mathrm{ms}$ [9] isomer were measured to be $<2 \%$ and $18(5) \%$, respectively, by observation of their $511 \mathrm{keV}$ (from internal pair creation) and $814 \mathrm{keV}$ decay $\gamma$ rays in two coaxial Ge detectors at the plastic scintillator beam stop.

Photons which were emitted in flight and in coincidence with the scattered nuclei were detected in the segmented
Ge clover detectors. Each Ge crystal representing a "leaf" of the clover is longitudinally segmented in four quadrants, reducing the angular acceptance of the individual detector elements. The use of the electronic segmentation of the Ge detectors reduces the Doppler broadening by about $40 \%$, leading to an energy resolution (FWHM) of $65 \mathrm{keV}$ at $1 \mathrm{MeV}$. The add-back mode has been used for the detection of the high-energy $\gamma$-ray transitions, increasing the efficiency $\left(\epsilon_{\gamma}\right)$ by $40 \%$ for a $\gamma$ ray at $2.033 \mathrm{MeV}$, resulting into $\epsilon_{\gamma}=3.4 \%$ [16]. This technique consists of adding the signals corresponding to events where a $\gamma$ ray has been scattered between adjacent leaves of the clover. The Doppler-corrected spectra for the three studied nuclei are shown in Fig. 1(a)-1(c). These spectra clearly show photopeaks associated with the Coulomb excitation of ${ }^{70} \mathrm{Zn}$ [17], ${ }^{66} \mathrm{Ni}$, and ${ }^{68} \mathrm{Ni}$ [18]. For ${ }^{68} \mathrm{Ni}$ no other $\gamma$ ray was observed besides the $2033 \mathrm{keV}$ line; the Coulomb excitation contribution of the $5^{-}$isomer was found to be negligible. The number of $\gamma$ rays $\left(N_{\gamma}\right)$ in the peaks is given in Table I. In the non-Doppler-corrected spectrum of ${ }^{68} \mathrm{Ni}$ [Fig. 1(d)], the peak at $2033 \mathrm{keV}$ is not visible anymore. The $\gamma$ line at $2.614 \mathrm{MeV}$ corresponds to the deexcitation of the $3^{-}$octupole state in the ${ }^{208} \mathrm{~Pb}$ target nuclei. As the segments of the clover detector are at four different angles, this $\gamma$ line emitted at rest is split into four peaks [Fig. 1(b)] when the Doppler-shift correction is applied.

The $B(E 2)$ values are determined in two ways: first, by normalization to the known $B(E 2)$ value of $1600(140) e^{2} \mathrm{fm}^{4}$ reported for ${ }^{70} \mathrm{Zn}$ in Ref. [17] and second, by an evaluation of the $\sigma_{0^{+} \rightarrow 2^{+}}$cross section taking into account the total number of nuclei, $N_{n}$, impinging on the target and the number of nuclei scattered in the $\mathrm{Si}$ detectors at angles between $1.5^{\circ}$ and $6.5^{\circ}$. Both the size of the secondary beam and the angular straggling in the target contribute to widening the angular distribution of the outcoming nuclei. The subsequent geometrical losses in the Si detectors, which amount to $28 \% \pm 2 \%$ for all nuclei, have been taken into account using a Monte Carlo simulation [16]. Cross sections have been rescaled according to these effects. Absolute $B(E 2)$ values have been derived by optical potential calculations with the use of the ECIS code [19] and experimental cross sections $\sigma_{0^{+} \rightarrow 2^{+}}$. The agreement between the absolute and relative $B(E 2)$ is very good (see Table I), giving confidence in the determination of the $B(E 2)$ values.

The behavior of the $B(E 2)$ values (Fig. 2) in the $\mathrm{Ni}$ isotopic chain provides a clear illustration of the structural evolution between the two magic nuclei ${ }^{56} \mathrm{Ni}$ and ${ }^{68} \mathrm{Ni}$. As neutrons are added to ${ }^{56} \mathrm{Ni}$, collectivity is gradually increased in the $p_{3 / 2} f_{5 / 2} p_{1 / 2}$ shell-model space until the number of active particles reaches a maximum value at middistance between $N=28$ and $N=40$. While filling the $f p$ orbits up to $N=40$, the amount of quadrupole excitations is drastically reduced to a value which is about 3 times smaller than that of ${ }^{56} \mathrm{Ni}$. [In Weisskopf units, the $B(E 2)$ 's of ${ }^{56} \mathrm{Ni}$ and ${ }^{68} \mathrm{Ni}$ are $9.4(1.5)$ and 3.2(7), 

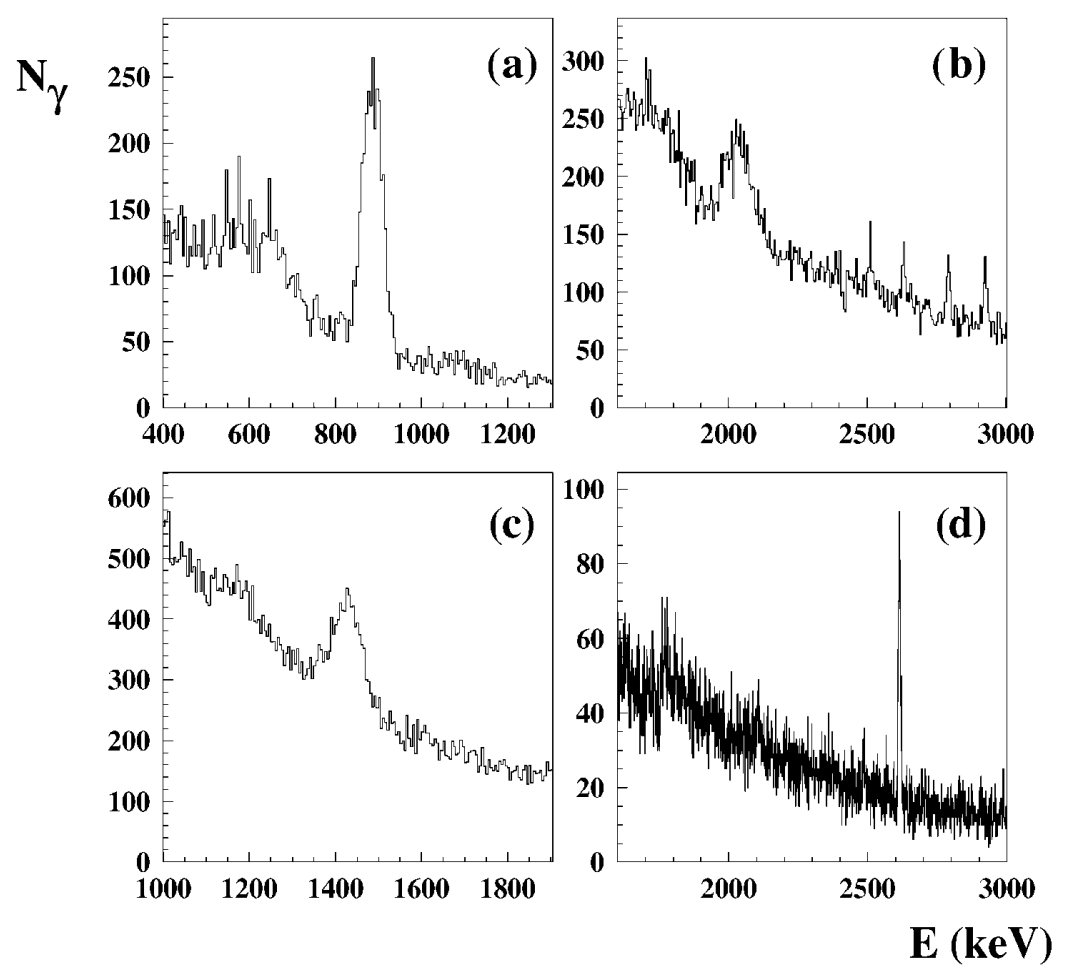

FIG. 1. $\quad \gamma$-energy spectra obtained in the Ge-clover detectors from the Coulomb excitation of ${ }^{70} \mathrm{Zn}(\mathrm{a}),{ }^{66} \mathrm{Ni}(\mathrm{c})$, and ${ }^{68} \mathrm{Ni}(\mathrm{b}),(\mathrm{d})$. Spectra (a)-(c) are Doppler corrected; (d) is not.

respectively.] This unexpected feature originates from the combined effects of a relatively large $B(E 2)$ of ${ }^{56} \mathrm{Ni}$ $[20,21]$ and an especially low value of ${ }^{68} \mathrm{Ni}$. The large $B(E 2)$ value of ${ }^{56} \mathrm{Ni}$ is due to the wide possibility of making particle-hole quadrupole excitations across $N, Z=28$ in the $f p$ shell for protons and neutrons [20] and a consequence of a strong proton-neutron interaction characterizing $N=Z$ nuclei $[22,23]$. The low $B(E 2)$ of ${ }^{68} \mathrm{Ni}$ is commonly assumed to be the signature of a doubly magic nucleus. Its value is comparable to the cases of the doubly magic nuclei ${ }^{16} \mathrm{O}$ [3.3(3) W.u.], ${ }^{40} \mathrm{Ca}$ [2.3(4) W.u.], and ${ }^{48} \mathrm{Ca}$ [1.6(5) W.u.]. This apparent indication of a shell gap is at variance with the observed two-neutron separation energies and their differences [24]. The hindrance of $2^{+}$excitations across the $N=40$ "shell" can be explained by a change of parity going from the $f p$ into the $g$ orbitals [25].

TABLE I. $N_{n}$, number of nuclei impinging onto the target. $v / c$, mean velocity in the middle of the target. $N_{\gamma}$, number of $\gamma$ rays due to the Coulomb excitation of ${ }^{70} \mathrm{Zn}$ and ${ }^{66,68} \mathrm{Ni}$ detected. $\sigma_{0^{+} \rightarrow 2^{+}}$and $B(E 2)_{\text {abs,rel }}$, cross section and absolute or relative $B(E 2)$ values for the $0^{+} \rightarrow 2^{+}$Coulomb excitation.

\begin{tabular}{ccccccc}
\hline \hline Isotope & $\begin{array}{c}N_{n} \\
\left(10^{9}\right)\end{array}$ & $v / c$ & $N_{\gamma}$ & $\begin{array}{c}\sigma_{0^{+} \rightarrow 2^{+}} \\
(\mathrm{mb})\end{array}$ & $\begin{array}{c}B(E 2)_{\mathrm{abs}} \\
\left(e^{2} f m^{4}\right)\end{array}$ & $\begin{array}{c}B(E 2)_{\mathrm{rel}} \\
\left(e^{2} f m^{4}\right)\end{array}$ \\
\hline${ }^{70} \mathrm{Zn}$ & 0.15 & 0.30 & 2790 & $730(120)$ & $1640(280)$ & 1600 \\
${ }^{66} \mathrm{Ni}$ & 0.94 & 0.29 & 4770 & $270(45)$ & $600(100)$ & $650(90)$ \\
${ }^{68} \mathrm{Ni}$ & 2.0 & 0.28 & 2690 & $105(25)$ & $255(60)$ & $280(60)$ \\
\hline \hline
\end{tabular}

In order to understand this and the trend of the $B(E 2)$ values observed in the $\mathrm{Ni}$ isotopes in more detail, we have performed shell-model calculations. It is impossible to consider the full $\mathrm{fpg}_{9 / 2}$ valence space with conventional diagonalization techniques, and, moreover, this latter space would generate spurious center of mass admixtures. Therefore, we have chosen to consider a ${ }^{48} \mathrm{Ca}$ core (more accurately, a ${ }^{40} \mathrm{Ca}$ core with $8 f_{7 / 2}$ frozen neutrons) with $f p\left(f_{7 / 2} p_{3 / 2} p_{1 / 2} f_{5 / 2}\right)$ active orbitals for protons and

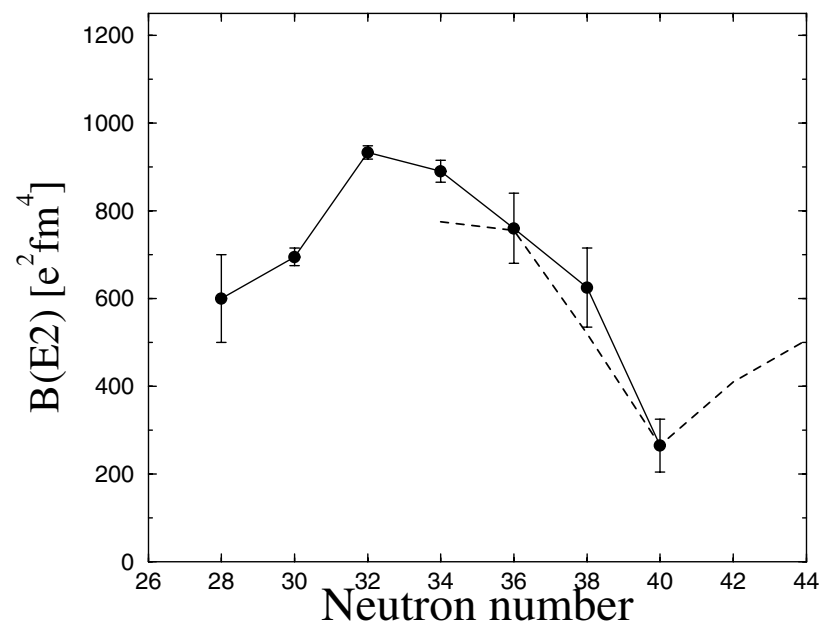

FIG. 2. Experimental $B\left(E 2 ; 0^{+} \rightarrow 2^{+}\right)$values in units of $e^{2} \mathrm{fm}^{4}$ for ${ }^{56} \mathrm{Ni}$ to ${ }^{68} \mathrm{Ni}$ nuclei are shown by filled circles. The dashed line corresponds to shell-model calculations. 
$p_{3 / 2} p_{1 / 2} f_{5 / 2}$ and $g_{9 / 2}$ (further on called $r g$ ) active orbitals for neutrons. An interaction was built for this space using $f p$ two-body matrix elements (TBME) from Ref. [26] and $r g$ TBME from Ref. [27]. For the common active orbitals in these subspaces, we kept the common matrix elements [27]. As the latter interaction $(r g)$ was defined for a ${ }^{56} \mathrm{Ni}$ core, a scaling factor of $A^{-1 / 3}$ amplitude was applied to take into account the change of radius between the ${ }^{40} \mathrm{Ca}$ and ${ }^{56} \mathrm{Ni}$ cores. As protons are added in the $f_{7 / 2}$ shell, the excitation energy of the $9 / 2^{+}$state is decreasing due to the attracting interaction $\pi f_{7 / 2} \nu g_{9 / 2}$. Therefore, we have taken the remaining $f_{7 / 2} g_{9 / 2}$ TBME from Ref. [28], fixed the unknown single particle energy $\epsilon_{g_{9 / 2}}$ to $9 \mathrm{MeV}$ in ${ }^{41} \mathrm{Ca}$ and set the $V_{f_{7 / 2} g_{9 / 2}}$ monopole strength in order to reproduce the excitation energy of the $9 / 2^{+}$state in ${ }^{57} \mathrm{Ni}$ at the zero-particle zero-hole $(0 p-0 h)$ level.

The ${ }^{64-74} \mathrm{Ni}$ isotopes were computed to study their behavior while crossing $N=40$. A polarization charge of $0.5 e$ for both protons and neutrons was used. Results are compiled in Table II and shown in Fig. 2. They correspond to a truncation of the valence space up to a total of six particle excitations from the $f_{7 / 2}$ orbital to the upper $f p$ orbitals for protons and from the upper neutron $f p$ orbitals to the $g_{9 / 2}$ orbital. We have checked that convergence was achieved for the observables considered. The calculation is able to reproduce the "bell-shape" behavior of the $E 2$ rates along the nickel isotopes as observed earlier up to $N=36$ [29]. It is found that proton core excitation from the $f_{7 / 2}$ orbit is responsible for more than $80 \%$ of the $B(E 2)$ value. The analysis of the wave functions shows a maximum of excitations from the neutron $f_{5 / 2}$ and $p_{1 / 2}$ shells to the $g_{9 / 2}$ orbital at $N=40$. This is inferred from the $g_{9 / 2}$ occupation number, which is listed in Table II as $\left\langle n_{9 / 2}\right\rangle_{\text {extra }}$, the difference to the normal occupation, i.e., zero up to $N=40$ and $N-40$ above. Correspondingly, the content of $(p, f)$ neutrons in the wave function shows a minimum at $N=40$. This is due to pairing correlations, which in ${ }^{68} \mathrm{Ni}$ gives rise to a mixed character of a closed shell and a superfluid nucleus. The constancy of the $B(E 2)$ values predicted beyond $N=40$ close to the small value observed for ${ }^{68} \mathrm{Ni}$ can be traced back to the limited possibility to generate $E 2$ strength as $n p$ - $n h$ excitations across $N=40$ for odd $n$ cannot contribute due to parity conservation and for even $n$ are domi-

TABLE II. Shell-model and experimental energies of the first excited state $E\left(2^{+}\right)[\mathrm{MeV}]$, calculated $B\left(E 2 \uparrow ; 0_{1}^{+} \rightarrow 2^{+}\right)$ $\left[e^{2} \mathrm{fm}^{4}\right]$, and the number $\left\langle n_{9 / 2}\right\rangle_{\text {extra }}$ of excessive neutrons occupying the $g_{9 / 2}$ orbit are compiled.

\begin{tabular}{lccccccc}
\hline \hline & ${ }^{62} \mathrm{Ni}$ & ${ }^{64} \mathrm{Ni}$ & ${ }^{66} \mathrm{Ni}$ & ${ }^{68} \mathrm{Ni}$ & ${ }^{70} \mathrm{Ni}$ & ${ }^{72} \mathrm{Ni}$ & ${ }^{74} \mathrm{Ni}$ \\
\hline$E\left(2^{+}\right)_{\text {calc }}$ & 1.11 & 1.24 & 1.49 & 1.73 & 1.50 & 1.42 & 1.33 \\
$E\left(2^{+}\right)_{\exp }$ & 1.173 & 1.346 & 1.425 & 2.033 & 1.259 & & \\
$B(E 2 \uparrow)_{\text {calc }}$ & 775 & 755 & 520 & 265 & 410 & 505 & 690 \\
$\left\langle n_{9 / 2}\right\rangle_{\text {extra }}$ & 0.24 & 0.43 & 0.67 & 1.19 & 0.73 & 0.45 & 0.27 \\
\hline \hline
\end{tabular}

nated by pair scattering, which smooths any occupation dependence of the $B(E 2)$ values.

In summary, the evidences for a possible $N=40$ shell gap are based on the following: ${ }^{68} \mathrm{Ni}$ has a large $2^{+}$excitation energy $E\left(2^{+}\right)=2.033 \mathrm{MeV}$ and a small $B\left(E 2 ; 0^{+} \rightarrow\right.$ $\left.2^{+}\right)=3.2(7)$ W.u. No shell gap is clearly evidenced from the two-neutron separation energies $S_{2 n}$ and their differences $\delta_{2 n}$ [24], which are free from pairing effects. The shell-model calculations reproduce these features, and the analysis of the underlying microscopic structure reveals that pair scattering of neutrons counteracts the magicity of the $N=40$ shell. The combined effect of neutron superfluidity and large proton $E 2$ polarizability leads to the erosion of the $N=40$ shell gap when adding or removing protons, as evident in the low $I^{\pi}=2^{+}$excitation energies in ${ }_{30}^{70} \mathrm{Zn}(0.885 \mathrm{MeV})$ and ${ }_{26}^{66} \mathrm{Fe}(0.573 \mathrm{MeV})$ [30]. The prospect of finding a $\mathrm{HO}$ shell closure in ${ }_{20}^{60} \mathrm{Ca}_{40}$ is very appealing. However, the expected gap between the $f_{5 / 2}$ and $g_{9 / 2}$ neutron orbitals will be reduced due to the missing monopole interaction $V_{f_{7 / 2} f_{5 / 2}}$, the $f_{7 / 2}$ proton orbital being unoccupied. This increases the tendency towards superfluidity.

We are thankful to the GANIL crew for the smooth running of the accelerator, to the EXOGAM Collaboration for providing the segmented-clover detectors, and to our colleagues from Russia for making the highly purified ${ }^{70} \mathrm{Zn}$ material available for the GANIL ion source. This work was partially supported by the following funding agencies: EU TMR ERBSMGECT 950036, OTKA Contract No. T30497, and GA AS Czech Republic A1048 605.

*Present address: Department of Physics and Astronomy, University of Manchester, Manchester M139PL, United Kingdom.

${ }^{\dagger}$ Present address: NSCL, Michigan State University, East Lansing, MI 48824.

[1] T. Otsuka et al., Phys. Rev. Lett. 87, 082502 (2001).

[2] J. Dobaczewski et al., Phys. Rev. Lett. 72, 981 (1994).

[3] M. Bender et al., in Nuclear Astrophysics: Proceedings of the International Workshop XXVI on Gross Properties of Nuclei and Nuclear Excitations, Hirschegg, Austria, 1998 (Gesellschaft für Schwerionenforschung, Darmstadt, 1998), p. 59.

[4] P. G. Reinhard et al., RIKEN Rev. 26, 23 (2000).

[5] H. Grawe and M. Lewitowicz, Nucl. Phys. A693, 116 (2001).

[6] L. Weissman et al., Phys. Rev. C 59, 2004 (1999).

[7] W. F. Mueller et al., Phys. Rev. Lett. 83, 3613 (1999).

[8] W. F. Mueller et al., Phys. Rev. C 61, 054308 (2000).

[9] R. Broda et al., Phys. Rev. Lett. 74, 868 (1995).

[10] R. Grzywacz et al., Phys. Rev. Lett. 81, 766 (1998).

[11] T. Ishii et al., Phys. Rev. Lett. 84, 39 (2000).

[12] B. Blank et al., Phys. Rev. Lett. 84, 1116 (2000).

[13] C. Engelmann et al., Z. Phys. A 352, 351 (1995).

[14] A. C. Mueller and R. Anne, Nucl. Instrum. Methods Phys. Res., Sect. B 70, 276 (1992). 
[15] S. Bouneau et al., Nucl. Instrum. Methods Phys. Res., Sect. A 443, 287 (2000).

[16] S. Leenhardt Ph.D. thesis, Orsay, 2000.

[17] P. H. Stelson and F. K. McGowan, Nucl. Phys. A32, 652 (1962).

[18] T. Pawlat et al., Nucl. Phys. A574, 623 (1994).

[19] J. Raynal, notes on Ecis94, Note CEA-N-2772 (unpublished).

[20] G. Kraus et al., Phys. Rev. Lett. 73, 1773 (1994).

[21] Y. Yanagisawa et al., in Exotic Nuclei and Atomic Masses, edited by Bradley M. Sherrill, David J. Morrissey, and Cary N. Davids, AIP Conf. Proc. No. 455 (AIP, Woodbury, NY, 1998), p. 610.

[22] T. Otsuka et al., Phys. Rev. Lett. 81, 1588 (1998).
[23] A. Poves, in Proceedings of the 6th International Spring Seminar on Nuclear Physics: Highlights of Modern Nuclear Structure, edited by A. Covello (World Scientific, Singapore, 1999), p. 129.

[24] A. M. Oros-Peusquens and P. F. Mantica, Nucl. Phys. A669, 81 (2001).

[25] H. Grawe et al., in Tours Symposium on Nuclear Physics IV, Tours 2000, edited by M. Arnould et al., AIP Conf. Proc. No. 561 (AIP, Melville, NY, 2001), p. 287.

[26] A. Poves et al., Nucl. Phys. A694, 157 (2001).

[27] F. Nowacki Ph.D. thesis, IRes, Strasbourg, 1996.

[28] S. Kahana et al., Phys. Rev. 180, 956 (1969).

[29] O. Kenn et al., Phys. Rev. C 63, 021302(R) (2001).

[30] M. Hannawald et al., Phys. Rev. Lett. 82, 1391 (1999). 